\title{
Structures and Generation of Current Filaments in Plasma Focus
}

\author{
V.Ya. Nikulin, S.P. Tsybenko* And A.A. ERISkin \\ P.N. Lebedev Physical Institute of the Russian Academy of Sciences, \\ 53 Leninskiy Pr., 119991 Moscow, Russia \\ Doi: 10.12693/APhysPolA.138.622 \\ *e-mail: sergeitsybenko@mail.ru
}

\begin{abstract}
The plasma focus filaments are investigated in the simple plasma model with the London current. The filament solutions depend on two main parameters - on the speed of filament motion (supersonic, subsonic and zero) and the filament radius which is less or more than the London penetration depth. The corrugation instability of rarefaction shock waves is studied in detail as the mechanism of the plasma focus filament generation.
\end{abstract}

topics: rarefaction shock wave, corrugation instability, filaments, London current

\section{Introduction}

The plasma focus $(\mathrm{PF})$ filaments are long-lived structures (their lifetime can be of the order of the discharge time [1]) and their diameter varies, mainly in the submillimeter range [2]. These structures are detected in the amount from several units to thousands in the PF discharges [3]. The filaments are generated at an insulator, and then propagate to the axis of a discharge chamber. At first, the representation about stationary filaments had been developed [4]. Recently, solutions have been proposed for the moving and stationary quasi-cylindrical filaments in the simple plasma model with the London current [5-7]. Here, a subsonic solution for submillimeter cylindrical structures is presented. Its radius is smaller than the London penetration depth. In addition, we discuss the mechanism for the PF filament generation which is associated with the corrugation instability of rarefaction shock waves (RSWs) [8, 9]. The reproducibility of emission characteristics may be improved and the intensity of PF radiation may increase if we induce filaments with metal inserts at the insulator.

\section{Main relations}

The basis of the developed theory consists of the dissipationless equations of two-fluid plasma hydrodynamics which result from the variational principle [10]. These equations were used, for example, in the theory of superconductivity by London [11] in which the relation for the London current $j$ has been established, $\boldsymbol{j}=-e^{2} n_{e} \boldsymbol{A} / m_{e} c$, where $n_{e}$ is the electron concentration and $\boldsymbol{A}$ is the vector potential. If the plasma hydrodynamic velocity is much less than the current velocity, one can proceed from the equations of two-fluid plasma hydrodynamics to the one-fluid model of quasi-neutral fully ionized plasma called the simple plasma model with the London current

$$
\begin{aligned}
& \frac{\partial \rho}{\partial t}+\nabla(\rho \boldsymbol{v})=0, \\
& \frac{\partial \boldsymbol{v}}{\partial t}+(\boldsymbol{v} \cdot \nabla) \boldsymbol{v}=-\frac{\nabla P}{\rho}-\frac{\partial \boldsymbol{v}}{2 m_{e} m_{i} c^{2}} \nabla \boldsymbol{A}^{2}, \\
& \nabla \times \nabla \times \boldsymbol{A}=-\frac{4 \pi Z e^{2}}{m_{e} m_{i} c^{2}} \rho \boldsymbol{A},
\end{aligned}
$$

where $Z e$ and $m_{i}$ are the ion charge and mass, $m_{e}$ is the electron mass, $c$ is the speed of light in a vacuum and $v$ is the plasma velocity. The plasma pressure $P$ is a function of density $\rho$. From the last equation of the system it follows that $\operatorname{div}(\rho \boldsymbol{A})=0$. Hence for $\boldsymbol{A} \perp \nabla \rho$ we obtain the Coulomb gauge for $\boldsymbol{A}$. We shall use the equations of plasma hydrodynamics when the characteristic scale of inhomogeneity of plasma and field is determined by the London penetration depth $L=c / \omega_{p}$, where $\omega_{p}$ is the electron plasma frequency.

\section{Cylindrical filaments}

The ratio of the diameter of a filament to its length is a small parameter that enables us to consider the filaments as quasi-cylindrical structures (or as cylindrical structures in the zero approximation with respect to the small parameter). We assume that the plasma is adiabatic, i.e., $P=P_{0}\left(\rho / \rho_{0}\right)^{5 / 3}$, where $P_{0}$ and $\rho_{0}$ are the plasma density and pressure around a filament, respectively. Here, filaments are considered as cylindrical structures that can move at a constant speed $D$ perpendicular to the axis of the filament. 
Then, the system is reduced to two equations for two independent variables $\rho$ and $A_{z}$, where $A_{z}$ is the component of the vector potential in cylindrical coordinates.

We proceed with dimensionless variables: $\rho / \rho_{0} \rightarrow \rho, \quad \omega_{p 0} r / c \rightarrow \tau, \quad D / v_{s 0} \rightarrow D, \quad$ and $Z e^{2} A_{z}^{2} / 2 m_{e} m_{i} c^{2} v_{s 0}^{2} \rightarrow a^{2}$, where $r$ is the cylindrical coordinate and $\omega_{p 0}$ and $v_{s 0}$ are the electron plasma frequency and the sound velocity in the plasma around the filament, respectively. Subsequently, Eqs. (1) take the following form:

$$
\begin{aligned}
& \frac{\mathrm{d}}{\tau \mathrm{d} \tau}\left(\tau \frac{\mathrm{d} a}{\mathrm{~d} \tau}\right)=\rho a, \\
& a^{2}=\frac{D^{2}}{2}\left(1-\frac{1}{\rho^{2}}\right)+\frac{3}{2}\left(1-\rho^{\frac{2}{3}}\right) .
\end{aligned}
$$

We seek a solution of the system (2) containing the tangential discontinuity. Note that for a moving cylindrical structure, there is a plasma flow through the tangential discontinuity.

Therefore, in this case one can use the approximate relation $P+\boldsymbol{B}^{2} / 8 \pi \approx$ constant, provided that the plasma flow is small through a discontinuity $\boldsymbol{B}=\nabla \times \boldsymbol{A}$. We introduce the coordinate $\tau_{* *}$ to indicate the location of the tangential discontinuity. For $\tau<\tau_{* *}$ in the solution for cylindrical structure, there is only a plasma pressure, since the magnetic field vanishes. We also introduce the coordinate $\tau_{*}\left(\tau_{* *}<\tau_{*}\right)$ to denote the discontinuity where the magnetic field reverses its direction. In this case, all other quantities remain continuous, for example, the plasma density $\rho_{*}=\rho\left(\tau_{*}\right)$. For dimensionless parameters, the condition for the smallness of the plasma flow through the tangential discontinuity takes the form of $D \rho_{* *}^{-1} \ll 1$, where $\rho_{* *}$ is the plasma density on the inner side of the discontinuity. The solutions are determined by the speed of movement of the filament $D$, the discontinuity position $\tau_{*}$, where the magnetic field changes its direction abruptly, and the density $\rho_{*}$ at this discontinuity. The solutions are rated in the order of two main parameters - on $D$ (supersonic, subsonic and zero) and filament radius $\tau_{*}$ (less or more than $L$ ). Incidentally, independently of a region where a solution is obtained (in any case of the six above regions), the forward discharge currents are flowing on the tangential discontinuity surface within the filament structure and the inverse induced currents are around the forward current. The induced currents magnetic field compensates the forward current magnetic field because the plasma with the London current is a perfect diamagnetic material.

Further, we demonstrate the subsonic solution $(D<1)$ with $\tau_{*}<L$ (see Figs. 1 and 2). All other cases have been submitted previously [6-8]. Here, we also show the images of moving and stationary submillimeter filaments of PF which have been obtained early in our laboratory by means of the image converter [12] (see Fig. 3). Figure 3 displays typical

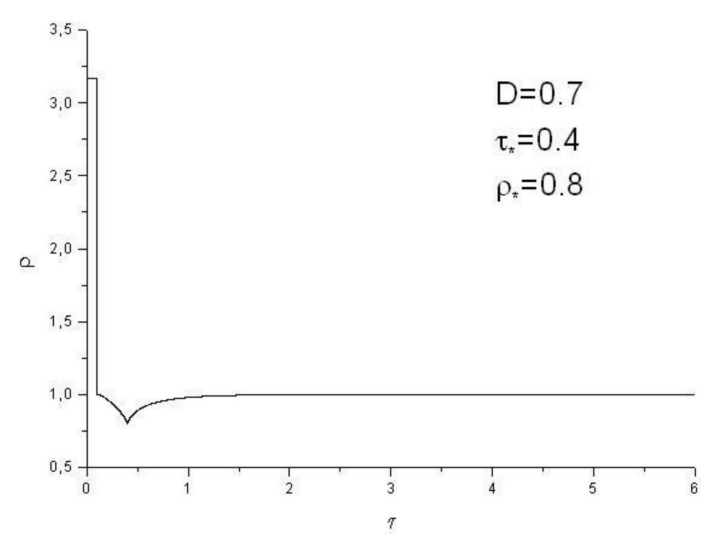

Fig. 1. The distribution of the plasma density along the radius in the filament.

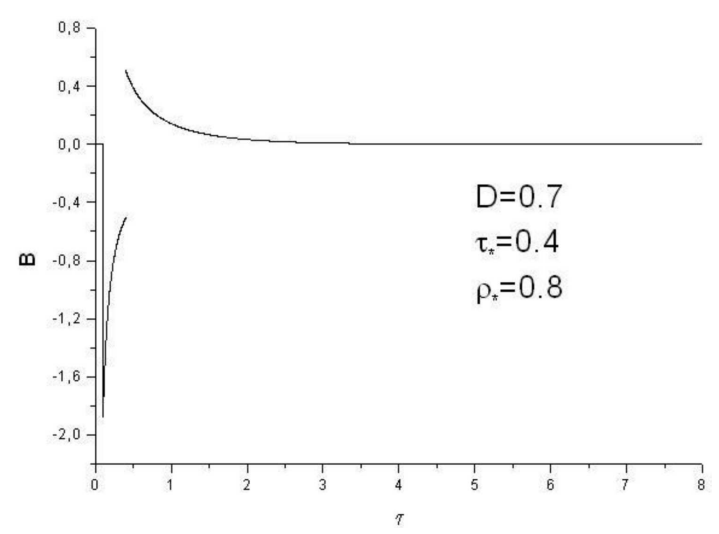

Fig. 2. The distribution of the magnetic field along the radius in the filament.

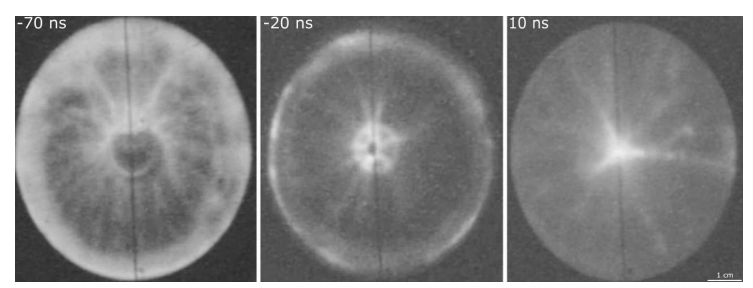

Fig. 3. Filamentation of the current sheath at different stages of the PF discharge.

discharge photos taken from the end of PF at different times near the instant at which a peculiarity in the current signal appears.

\section{Corrugation instability of RSW}

The simple model solution for RSW [10] is the current flowing over the discontinuity surface which propagates towards plasma with a higher density. This current generates a magnetic field on both sides of the discontinuity, with a larger magnetic field generated on the side with a higher plasma density. The magnetic field, in turn, induces reverse London currents on both sides of the gap. RSWs are supersonic as well as compression shock waves. 
To study the instability of RSW, we pass to the coordinate system that moves with the discontinuity. We assume that the plasma moves perpendicular to the discontinuity surface and study periodic perturbations of a plane RSW with a wave vector $\boldsymbol{k}$ perpendicular to the unit normal to the discontinuity surface $\boldsymbol{n}$. Below we consider the problem when the vectors $\boldsymbol{k}$ and $\boldsymbol{n}$ are perpendicular to the current flowing along the discontinuity surface (see Fig. 4). Based on the symmetry of the problem, we write the expression for the perturbation of the plasma velocity in the form $\delta \boldsymbol{v}=\delta v_{t} \boldsymbol{k} / k$, where $\delta v_{t}$ is the tangential velocity perturbation. Let us also consider perturbations of the vector potential $\boldsymbol{a}$ parallel to the unperturbed value of the vector potential $\boldsymbol{A}$ which, in turn, is parallel to the direction of the current flowing along the discontinuity surface. Note that the perturbation of an arbitrary parameter $\psi$ of the plasma or field is a plane wave which is conveniently written as $\psi \sim \exp (-\mathrm{i} \omega t+\mathrm{i}(\boldsymbol{k}+\boldsymbol{\varkappa} \boldsymbol{n}) \boldsymbol{r})$, where $\varkappa$ is the component of the wave vector along the vector $\boldsymbol{n}$. Further, from the linearized system (1) we obtain the relation which connects $\omega$, $k$ and $\varkappa$ on each side of the discontinuity

$$
\begin{aligned}
& \left(\omega-v_{n} \varkappa\right)^{2}= \\
& \quad\left[v_{S}^{2}-\frac{2 U}{\rho} \frac{1}{1+L^{2}\left(k^{2}+\varkappa^{2}\right)}\right]\left(k^{2}+\varkappa^{2}\right),
\end{aligned}
$$

where $v_{n}$ is the unperturbed plasma velocity normal to the discontinuity surface and $U=2 \pi j^{2} / \omega_{p}^{2}$ is the self-energy density of currents which are induced on the discontinuity sides.

For the unperturbed values of the plasma and field and for their perturbations on the sides of the RSW, we obtain from the system (1) the relation for the momentum flux continuity

$$
\begin{aligned}
& \left\{P+\rho v_{n}^{2}+\frac{B_{t}^{2}}{8 \pi}\right\}=0, \\
& \left\{\delta P+\delta \rho v_{n}^{2}+\frac{B_{t} b_{t}}{4 \pi}\right\}=0, \\
& \left\{\rho v_{n} \delta v_{t}-\frac{B_{t} b_{n}}{4 \pi}\right\}=0 .
\end{aligned}
$$

Braces denote the difference in parameter values on the sides of the discontinuity. The indices $t$ and $n$ denote the tangential and normal components of the vectors. Here $\delta P$ and $\delta \rho$ are perturbations of pressure and plasma density and $b_{n}$ and $b_{t}$ are the components of magnetic induction perturbation. We write the equation of motion from the system (1) for perturbations of the plasma and field as follows:

$$
\begin{gathered}
\rho \frac{\partial \delta v_{t}}{\partial t}=-\frac{\partial}{\partial \eta}\left(\delta P-\frac{B_{t} \delta b_{t}}{4 \pi}\right) \\
-\frac{\partial}{\partial \xi}\left(\rho v_{n} \delta v_{t}-\frac{B_{t} b_{n}}{4 \pi}\right),
\end{gathered}
$$

where $\eta=\boldsymbol{k r} / k$ and $\xi=\boldsymbol{n} \boldsymbol{r}$ are the coordinates along the vectors $\boldsymbol{k}$ and $\boldsymbol{n}$, respectively.

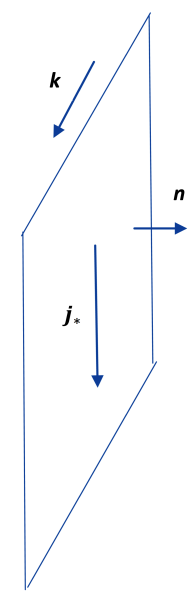

Fig. 4. The wave vector $\boldsymbol{k}$, the normal to the plane RSW $\boldsymbol{n}$ and the surface current density $\boldsymbol{j}_{*}$.

After setting perturbations, (5) is reduced to the following:

$$
\omega \rho \delta v_{t}=k^{2}\left(2 v_{S}^{2}+v_{n}^{2}\right) \frac{\rho \delta v_{t}}{\omega-\varkappa v_{n}} .
$$

We define $\delta v_{t}$ through the displacement of the plasma perturbation $\tau$ along the vector $\boldsymbol{k}$ as:

$$
\frac{\partial \tau}{\partial t}+v_{n} \frac{\partial \tau}{\partial \xi}=\delta v_{t},
$$

where $\tau \sim \exp (-\mathrm{i} \omega t+\mathrm{i} k \eta+\mathrm{i} \varkappa \xi)$. Then, we obtain from (6) the dispersion relation for the corrugation instability of RSW in the following form:

$$
\omega\left(\omega-\varkappa v_{n}\right) \rho+k^{2}\left(\frac{7}{3} \rho v_{n}^{2}+\frac{5}{3} U\right)=0 .
$$

We study the case of a strong RSW when $\rho_{1} \gg \rho_{2}$ and $U_{1} \gg U_{2}$, where indices 1 and 2 denote the values on the discontinuity sides as well as $U_{1} \gg \rho_{1} v_{1}^{2}$, $\rho_{2} v_{2}^{2}$ and $|\omega| \gg \varkappa_{1} v_{1}$. As a result, the dispersion relation (8) will be as follows:

$$
\omega^{2} \rho_{1}+\frac{5}{3} U_{1} k^{2}=0
$$

Then, for the increment of the corrugation instability of a strong RSW, we write the expression

$$
\nu=k \sqrt{\frac{5}{3} U_{1} \rho_{1}} .
$$

Now let us see that the inequality $|\omega| \gg \varkappa_{1} v_{1}$ is valid for a strong RSW. From (3) for $k \approx \omega_{p} / c$ we obtain that $\varkappa_{1}= \pm 2 \omega_{p} / c$. As a result, the condition $|\omega| \gg \varkappa_{1} v_{1}$ can be transformed to the inequality $U_{1}^{1 / 2} \gg \rho_{1}^{1 / 2} v_{1}$ which is performed for a strong RSW.

The theory of the corrugation instability of a strong plane RSW may be extended to the case of a cylindrical RSW (see Fig. 5) taking into account that $L \ll R$ and considering perturbations with $\varkappa_{1} L \gtrsim 1$. Here $R$ is the radius of the cylindrical RSW determined by the radius of the insulator. The generation of PF current filaments is associated with the corrugation instability of a strong cylindrical RSW. Moreover, the filament 


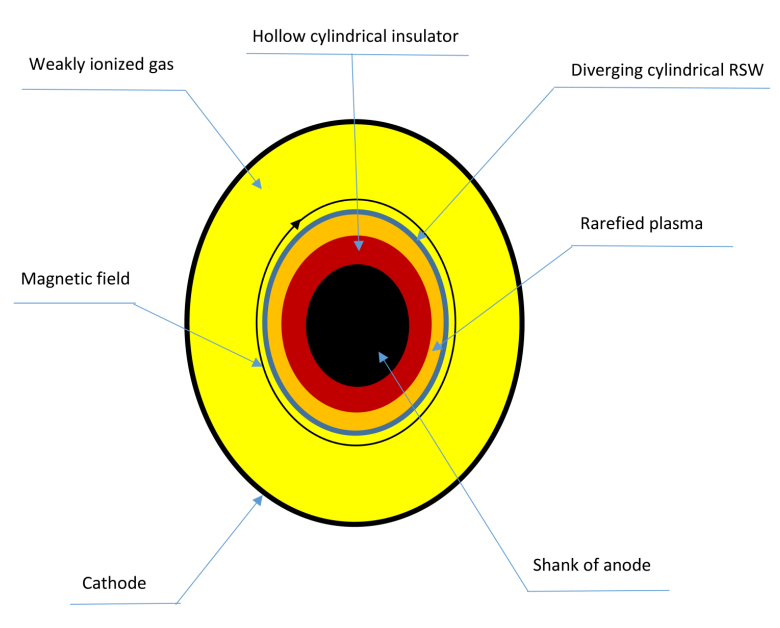

Fig. 5. The image of the PF chamber crosssection.

formation period $t_{F}$ is determined by the corrugation instability increment $\nu$. An earlier evaluation of the filament formation period [8] yielded a value of $t_{F}<100 \mathrm{~ns}$.

In order to achieve the induced generation of $\mathrm{PF}$ filaments, it is necessary to use metal inserts in the places where the electrodes contact the insulator [13]. In this case, the inserts must have a serrated edge to stimulate the corrugation instability with a given modulation scale that matches the size of the teeth on the inserts. It is experimentally possible to determine the optimal number of teeth on the inserts which sets the number of PF filaments. The optimal number of teeth for PF with an insulator radius of $1 \mathrm{~cm}$ is expected to be about 100 or more [8].

\section{Conclusions}

The current filament generation mechanism in PF associated with the development of the corrugation instability of the strong RSW is presented. For this, the simple plasma model with the London current is used. In the same model, the structures of current filaments are investigated. The induced filamentation of the PF current sheath with the help of special-shaped metal inserts is proposed.

\section{Acknowledgments}

This study was supported by the Russian Science Foundation \#16-12-10351.

\section{References}

[1] W. Sadowski, H. Herold, H. Schmidt, M. Shakhatre, Phys. Lett. A 105, 117 (1984).

[2] A. Bernard, H. Bruzzone, P. Choi et al., J. Moscow Phys. Soc. 8, 93 (1998).

[3] V. Nardi, W.H. Bostick, J. Feugeas, W. Prior, Phys. Rev. A 22, 2211 (1980).

[4] V. Nardi, Phys. Rev. Lett. 25, 718 (1970).

[5] V.Ya. Nikulin, S.A. Startsev, S.P. Tsybenko, Bull. Lebedev Phys. Inst. 42, 133 (2015).

[6] V.Ya. Nikulin, S.A. Startsev, S.P. Tsybenko, Bull. Lebedev Phys. Inst. 43, 345 (2016).

[7] V.Ya. Nikulin, S.A. Startsev, S.P. Tsybenko, IOP Conf. Series J. Phys. Conf. Series 907, 012024 (2017).

[8] S.P. Tsybenko, A.E. Gurei, Bull. Lebedev Phys. Inst. 46, 157 (2019).

[9] V.Ya. Nikulin, S.P. Tsybenko, A.E. Gurei, Bull. Lebedev Phys. Inst. 44, 163 (2017).

[10] V.Ya. Nikulin, S.P. Tsybenko, Phys. Scr. 55, 90 (1997).

[11] F. London, Superfluids, Wiley, New York 1950.

[12] V.Ya. Nikulin, S.N. Polukhin, A.A. Tikhomirov, Plasma Phys. Rep. 31, 591 (2005).

[13] N.V. Filippov, Fiz. Plazmy 9, 25 (1983) [Sov. J. Plasma Phys. 8, 24 (1983)]. 